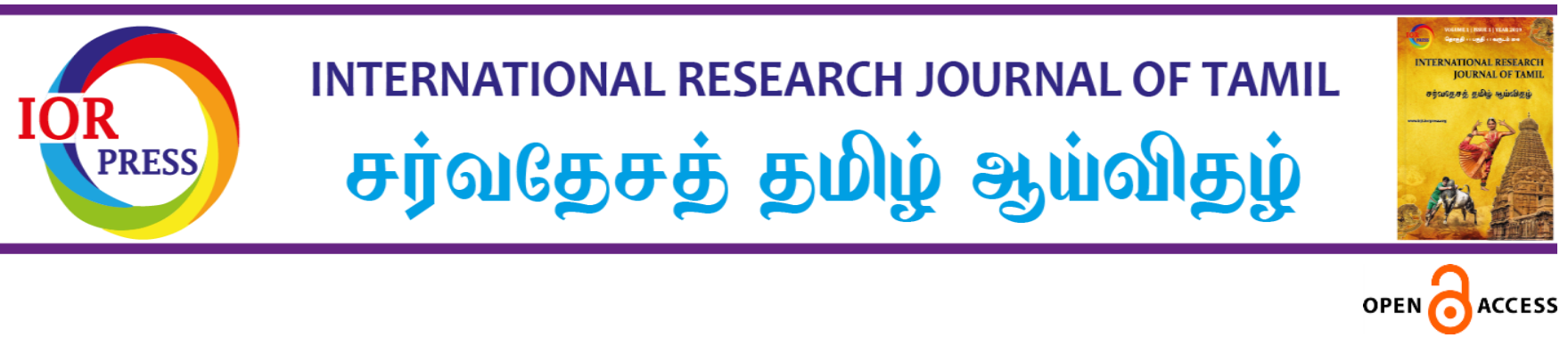

வள்ளுவம் வலியுறுத்தும் மக்கள் நல அரசு

இரா. வெங்கடேஷ் அ, •

அ அண்ணா பொதுவாழ்வியல் மையம், சென்னைப் பல்கலைக்கழகம், சென்னை-600005, தமிழ்நாடு, இந்தியா.

\title{
Emphasis of Welfare State in Valluvam
}

\author{
R. Venkatesh ${ }^{\text {a }},{ }^{*}$ (D) \\ a Anna Centre for Public Affairs, University of Madras, Chennai-600005, Tamil Nadu, India
}

* Corresponding Author: rvsh76@gmail.com

Received: 04-11-2020

Revised: 09-02-2021

Accepted: $12-02-2021$

Published: 13-03-2021

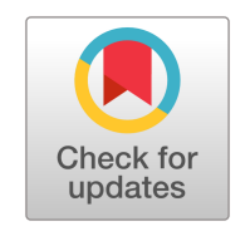

\begin{abstract}
The relationship between literature and politics is a reciprocal one. The core theme of literature makes a positive impact on individual and society. Literature was considered as a powerful tool to explore the moral, ethical and social values which brought notable changes in the socio - political sphere. In this context, Thirukkural is most important universally accepted literature as it reveals the ethical values of politics and administrative system found in the erstwhile Tamil society like elements of state, fortification, excellence of army, factors for good governance, qualities of a king, minister, avoidance of faults, etc. Thirukkural is a timeless Tamil Classic written approximately 2,000 years ago by the great thinker and philosopher - Saint Thiruvalluvar. Ethical and political principles of a very high standard are expressed in the Thirukkural in a lucid and precise manner which also applicable to the present day democratic governance. Out of the three, the second part 'Porul', (Wealth) is a treatise on political philosophy and administrative practices as well as on economy. It is directly addressed to the leader or the Ruler. As such this paper attempts to highlight the administrative thoughts of Thiruvalluvar in the context of good governance and welfare politics that existed in ancient Tamil society.
\end{abstract}

Keywords: Administration, Politics, Welfare State, Good Governance, Democracy.

\section{ஆசிரியர் குறிப்பு}

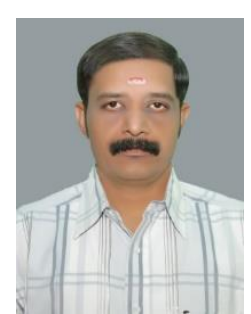

முனைவர் இரா. வெங்கடேஷ், சென்னைப் பல்கலைக்கழகத்தில் அண்ணா பொது வாழ்வியல் துறையில் உதவிப் பேராசிரியராகப் பணியாற்றி வருகின்றார். அரசியல் தத்துவங்கள் மற்றும் ஆட்சியியல் கோட்பாடுகளைத் தமிழ் இலக்கிய, இலக்கண ஆதாரங்களுடன் ஒப்பிட்டு திறனாய்வு செய்வதில் மிகுந்த ஈடுபாடு கொண்டவர். இவர் தமிழார்வம் நிரம்பப் பெற்ற நற்றமிழ் ஆர்வலராக அறியப்படுகின்றார். நாளிதழ்கள் மற்றும் ஆய்விதழ்களில் இவரது கட்டுரைகள் தொடர்ந்து வெளிவருகின்றன.

\section{முன்னுரை}

பொதுவாக மனிதன் கூடிவாழும் இயல்பு படைத்தவன் அவ்வாறு கூடிவாழும் போது பல்வேறு அம்சங்களை உள்ளடக்கிய சட்ட திட்டங்களும், கட்டுப்பாடுகளும் உருவாகின்றன. அத்தகைய 
கட்டுப்பாடுகள் சமுதாய வாழ்வை நெறிப்படுத்துகின்றன. நெறிப்பட்ட வாழ்க்கையை அமைத்துத் தரும் காரணிகளுள் ஒன்று அரசியல். அரசியலை வழிநடத்திட அரசு எனும் அமைப்புத் தேவைப்படுகின்றது. அரசியலின் அடிப்படை அம்சமான அரசு மனித ஒழுக்கங்களை, வாழ்க்கை முறைமைகளை சட்டதிட்டங்களின் படி நெறிப்படுத்துகின்றது. மனிதர்களின் வாழ்வை நிர்ணயிக்கும் அரசு எனும் அமைப்புதான் மக்களின் வாழ்க்கைத்தர மேம்பாட்டிற்குத் துணைநிற்கின்றது. அந்த வகையில் அரசியல் நுட்பங்களை, ஆட்சியியல் நெறிகளை நன்குணர்ந்து எடுத்துரைத்தவர் வள்ளுவராவார்.

தொன்மைச் சிறப்பு வாய்ந்த இலக்கியங்கள் பல அரசியல் மற்றும் ஆட்சியியல் சிந்தனைகளை எடுத்தியம்புகின்றது. அரசு, அரசன், ஆட்சிமுறை, போர், செங்கோல், குணநலன் எனப் பல்வேறு ஆட்சியியல் கூறுகள் சங்க இலக்கியத்தில் பேசப்படுகின்றன. அரசனின் குணநலன்களைப் பாடுவதுடன் அறத்தின் அவசியத்தை வலியுறுத்தும் நோக்கில் சங்கப் பாடல்கள் அமைந்துள்ளன. ஆட்சியியல் அறத்தை வலியுறுத்தும் ஐம்பெரும் காப்பியங்களுள் ஒன்றான சிலப்பதிகாரம் சட்டத்தின் முன் அனைவரும் சமம் என்ற சட்டவியலின் அடிப்படைக் கோட்பாட்டினை வெளிப்படுத்துகின்றது. இதுபோலப் பல்வேறு இலக்கியங்கள் வாழ்வின் அம்சங்களைப் பிரதிபலித்தாலும் அவற்றுள் முதன்மையானதாகத் திருக்குறள் அறியப்படுகின்றது. காரணம் திருக்குறள் ஒன்றுதான் அ அறத்துடன் கூடிய அரசியல் மற்றும் ஆட்சியியல் சிந்தனைகளை இச்சமூகத்தின் மேன்மைக்காகவும், மக்களின் வாழ்க்கை உயர்விற்காகவும் எடுத்துச் சொல்கின்றது.

நம் அரசியலமைப்பின் தனிக்கூறுகளான சமயச்சார்பின்மை, வேற்றுமையில் ஒற்றுமை, உரிமைகள், அரசின் குறிக்கோள்கள், ஆட்சிச் செங்கோன்மைகள், ஆட்சியாளரின் செயல்திறன், நேர்மை தவறாப் பண்பு, சமத்துவம், சட்டநேர்மை உள்ளிட்ட பல்வேறு சிறப்புகளை எளிய முறையில் மக்களுக்கு உணர்த்திய இணையற்ற நூல் திருக்குறள். சாதாரணக் குடிமகன் முதல் சர்வ வல்லமை பொருந்திய அரசன் வரை அனைவருக்கும் முறையாக வழிகாட்டிய பெருமை திருக்குறளுக்கும் வள்ளுவருக்கும் உண்டு.

உன்னதமான வாழ்வியல் கருத்துக்களை உலகிற்கு உரக்கச் சொன் வள்ளுவர் நடைமுறைக்குப் பொருந்தும் வகையிலும் அனுபவ வாழ்விற்கு ஏற்ற வகையிலும் சட்ட, நிர்வாக மற்றும் நீதி நெறிமுறைகளை வழங்கினார். இதில் அறிவிற்குப் பொருத்தமற்ற, யதார்த்த வாழ்விற்குப் பொருந்தாத நெறிகள் எதுவுமில்லை எனலாம். திருக்குறள் ஓர் அறநூல் எனப் போற்றப்பட்டாலும் அறம் சார் ஆட்சியியல் சிந்தனைகள் ஏராளம் உண்டு. ஒரு நாடு எவ்வாறு இருக்க வேண்டும்? நாட்டினை ஆட்சி செய்கின்ற ஆட்சியாளர் (அரசன்) எங்ஙனம் ஆட்சி நடத்த வேண்டும்? நல்லாட்சிக்கான கூறுகள் எவை? சட்டத்தின் ஆட்சியை நிலைநாட்டுவதற்கு அவனுக்கு எத்தகைய தகுதிகள் இருத்தல் வேண்டும்? தவறு நடக்கையில் நேர்மை தவறாது, சார்பின்றி எவ்வாறு நீதி வழங்க வேண்டும்? என்பன போன்ற கருத்துக்களை இரண்டாயிரம் ஆண்டுகளுக்கு முன் தந்து நிற்பவர் வள்ளுவராவார். காலம் முந்தி கருத்துக்களைத் தந்தாலும் அவை காலம் கடந்து இன்றைய ஆட்சி முறைமைக்கும் பொருந்துவது பெருமைக்குரியதாக உள்ளது.

\section{வள்ளுவர் - தத்துவவாதிகள்}

கௌடில்யரால் எழுதப்பட்ட முதல் அரசியல் நூலான அர்த்தசாஸ்திரம் அரசியல் அரிச்சுவடிகளைக் கோடிட்டுக் காட்டினாலும் அரசியல் பொருளாதாரம் பற்றித் தான் பெரிதும் விளக்குகின்றது.

T. H. மார்ஷல் எனும் அறிஞர் குடிமக்களின் சமூக, பொருளாதார, அரசியல் உரிமைகளைப் பேணும் வகையில் செயல்படுவது தான் மக்கள் நல அரசு என வலியுறுத்துகின்றார்.

மௌரியப் பேரரசர் அசோகர் கி.மு. 3ஆம் நூற்றாண்டிலேயே தர்மத்தின்படி ஆட்சி நடத்துவது தான் மக்களாட்சிக்கு வலுச்சேர்க்கும் என்கிறார்.

கென்ட் எனும் அறிஞர் மக்கள் நல அரசு என்பது குடிமக்களின் வாழ்க்கைத் தர மேம்பாட்டிற்கான சமூக சேவைகளை வழங்குவது தான் என்கிறார். 
காந்தியக் கொள்கையான சர்வோதயா அனைவரின் நலனைப் பாதுகாப்பதே மக்கள் நல அரசின் முதன்மைக் கொள்கையாகும் என எடுத்துரைக்கின்றது.

எந்தவொரு செயலையும் ஆராயாமல் சீர்தூக்கிப் பார்க்காமல் சிந்தனையும் அறிவும் அறவே இன்றி ஆரவாரம் செய்வதல்ல மக்களாட்சி. நேர்மையும் நற்பண்பும் கொண்டவர்கள் போலித்தனமின்றி நிர்வாகத்தைச் செலுத்துவதுதான் மக்கள் நல அரசு. அதுதான் நேர்மையான மக்களாட்சி என்கிறார். அந்த அரசில் ஒவ்வொரு குடிமகனும் தனது பொறுப்புகளை உணர்ந்து நடந்து கொள்வது என்பது சாக்ரடீஸின் கண்ணோட்டமாகும்.

பிளேட்டோ எனும் கிரேக்கத் தத்துவஞானி தன்னுடைய குடியரசு என்னும் நூலில் ஒரு நாடு எப்படி இருக்க வேண்டும்? எவ்வாறு செயல்பட வேண்டும்? தலைவனின் (மன்னன்) தனிச் சிறப்புகள் என்ன? என்பது பற்றியும் மக்களிடம் அதிகாரம் இருப்பது தான் உண்மையான மக்களாட்சி என்றும் வரையறுத்துக் கூறுகின்றார்.

அரிஸ்டாட்டில் தான் எழுதிய அரசியல் என்ற நூலில் அரசியலமைப்பின் பல்வேறு பரிமாணங்களைப் பற்றி ஆதாரங்களுடன் பதிவு செய்திருப்பினும் மக்களாட்சி முறை தான் சிறந்தது என்கிறார். மேலம் அதன் தலைமைப் பொறுப்பை ஏற்பவரின் முதன்மையான தகுதிகளையும் சுட்டுகின்றார். நல்லொழுக்கம் நிறைந்த மனிதனே மக்கள் நல அரசை வழி நடத்திட முடியும் என்கிறார்.

நிக்கோலோ மாக்கியவல்லி எனும் மேலை நாட்டு அறிஞர் முடியரசாயினும் குடியரசாயினும் மக்கள் நலமாக வாழ வேண்டும். அது மக்கள் நன்மை கருதும் அரசாக இருக்க வேண்டும். அப்போது தான் நிலைத்து நிற்கும் என்கிறார்.

ஆனால் மேற்சொன்ன அறிஞர்கள் அனைவரின் கருத்துக்களையும் தொகுத்து திருக்குறள் மக்களுக்காகச் செயல்படும் நிர்வாக அமைப்பு எத்தன்மை வாய்ந்ததாக இருத்தல் வேண்டும்? அரசியல் எப்படி இருக்க வேண்டும்? மக்கள் நல அரசின் கூறுகள், அரசை வழிநடத்தும் தலைவரின் தகுதிகள், அமைச்சரின் பண்புகள் போன்ற நிர்வாகத்தோடு தொடர்புடைய பல்வேறு அம்சங்களை விவாதிக்கிறது. அரசியல் மற்றும் ஆட்சியியலின் அனைத்துப் பரிமாணங்களையும் தெளிவுற எடுத்தியம்புவதால் வள்ளுவர் பிற தத்துவவாதிகளை விட அறநெறி அரசியலை வலியுறுத்துவதில் உயர்ந்து நிற்கின்றார் (Perumal, 1974; Thangayyan, 1950; Pope et. al., 1982).

\section{பொருட்பாலும் ஆட்சியியலும்}

நாட்டின் சிறந்த ஆட்சி முறையும் பாதுகாப்பான சமுதாயக் கட்டமைப்பும் மாண்பு நிறை மனித வாழ்விற்கு அடித்தளமிடும் காரணிகள் என்பதை பொருட்பால் எடுத்தியம்புகின்றது. எனவே தான் தனிமனிதனின், சமூகத்தின் மாண்புகளைப் பிரதிபலிக்கும் வள்ளுவம் உலக இலக்கியங்களே வியந்து போற்றும் ஆழமான, விரிவான ஒரு பன்முகக் கோவை என்றால் மிகையாகாது. மனிதன் மட்டுமல்ல அறநிலையை நிலைநாட்டுவதற்காகச் செயல்படும் ஆட்சியியல் அமைப்புகளும் சீரிய வழிகாட்டு நடைமுறைகளைப் பின்பற்ற வேண்டும் எனத் தெளிவாக உணர்த்துவதை அறிய முடிகின்றது. அதற்கான முன்னோட்டமாக அறம், பொருள், இன்பம் எனும் மூன்று பகுதிகளாகப் பிரித்து வாழ்வியலின் பொருளைத் திறம்பட நுட்பமாக எடுத்துரைத்தவர் வள்ளுவர்.

வள்ளுவத்தின் முப்பெரும் பிரிவுகளுள் பொருள் நடுவில் இடம் பெற்றுள்ளது. இப்பொருளை இரு விதங்களில் பொருள் கொள்ளலாம். பொருளீட்டல் - அதுவும் அறத்தால் பொருளீட்டி அப்பொருளால் இன்பம் நுகர்தலே வாழ்க்கை முறையாகும். பிறிதொரு கோணத்தில் பொருள் என்பதற்கு மக்களை உள்ளடக்கிய நிலம் என்ற பொருளும் உண்டு. பொருட்பாலில் உலக வாழ்க்கையின் புற இயல்புகளையும் நடைமுறைகளையும் அலசி ஆராயும் அனுபவம் நிறைந்த அறிஞராக வள்ளுவர் வெளிப்படுகின்றார். ஏழு பகுதிகள், எழுபது அதிகாரங்களை உள்ளடக்கிய பொருட்பாலில் அரசு(நிலம்), ஆட்சியியலின் சிறப்பம்சங்கள் (ஆள்திறன்), அரசனின் குணநலன்கள் (நிலத்தை ஆள்பவன்), பொருளாதாரம், குடிமக்களின் பண்புகள் போன்றவை கருப் பொருள்களாக 
உருப்பெற்றுள்ளன. மேலும் அறவழிப்பட்ட நாடு, அந்நாட்டின் நிர்வாக முறைகள், ஆட்சியாளனின் அறிவு, ஆற்றல், வீரம், அற உணர்வு போன்ற தகுதிகள் பற்றியும் குறிப்பால் உணர்த்துகின்றது. எனவே பொருட்பால் அரசியல், ஆட்சிமுறை, மக்கள் நலன் ஆகியவை மட்டுமல்லாமல் ஒழுங்கமை சமுதாய வாழ்விற்கான ஆட்சியியல் நுட்பங்களையும் ஒருங்கிணைத்துக் கூறுகின்றது. எல்லாவற்றிற்கும் மேலாக ஆட்சிக்கான இலக்கணங்களைப் பொதுமை நோக்கில் உணர்த்துவது இதன் தனிச் சிறப்பாகும் (Sankaravallinayagam, 2003).

\section{முடியாட்சியில் குடியாட்சி கண்ட வள்ளுவர்}

மக்களுக்காக, மக்களால், மக்களே தேர்ந்தெடுக்கும் ஆட்சி முறையே குடியாட்சி அல்லது மக்களாட்சி. மாறாக, அதிகாரங்கள் அனைத்தும் ஒருவரிடத்தே குவிந்து கிடக்கும் ஆட்சி முறை முடியாட்சி. சித்தாந்தத்தின் அடிப்படையில் ஒன்றுபட்டுள்ள ஒரு குழுவால் ஆளப்படும் ஆட்சி முறை குழுவாட்சி. மூன்றிலும் சிறந்தது குடியாட்சி தான். குடிமக்கள் தங்கள் அதிகாரத்தைப் பயன்படுத்திட வழிவகுக்கும் சிறந்த ஆட்சிமுறை.

ஆட்சிமுறை எதுவாயினும் சட்டமியற்றும் துறை, செயலாக்கத் துறை, நீதித் துறை ஆகிய மூன்று துறைகள் முக்கிய இடம் பெறுகின்றன. இம்மூன்று அதிகார நிலைகளும் முடியாட்சிக் காலத்தில் ஒருவரிடத்தே குவிந்திருந்தன. ஆனால் தற்போதைய மக்களாட்சிக் காலத்தில் அதிகாரம் பிரிந்து தனித்தனியே இயங்கினாலும் ஒன்றுக்கொன்று சார்புடன் செயல்படுகின்றது. எந்த ஆட்சியாயினும் அது மக்கள் நலனை முன்னிறுத்தும் ஆட்சி முறை தானே. ஆனால் மக்களே கண்ணீர் விடும் நிலையில் ஆட்சி இருந்தால் அது தேவையற்றது. அது அழிய வேண்டியது. இதை வள்ளுவர் நுட்பமாக

\section{அல்லற்பட்டு ஆற்றாது அழுத கண்ணீர்}

\section{செல்வத்தைத் தேய்க்கும் படை (குறள் - 555)}

எனக் கூறியுள்ளார். மக்கள் புரட்சிக்கு முன்னால் எதுவும் நிற்க முடியாது என்பதை முடியாட்சிக் காலத்தியே இயம்பிய வள்ளுவப் பேராசான் அரசியல் நுணுக்கம் தான் என்னே!

வள்ளுவர் முடியாட்சிக் காலத்தில் குடியாட்சி (மக்களாட்சி) பற்றிச் சிந்தித்த முதல் ஆட்சியியல் அறிஞர். அவரின் ஆட்சியியல் சிந்தனைகள் எவ்வாறு தற்போதைய நடைமுறைக்குப் பொருந்துகின்றது என்பது பற்றிக் காண்போம்.

\section{வள்ளுவ நாடு}

மனிதச் செயல்பாடுகளை, வாழ்க்கை முறைமைகளை விதிமுறைகளுக்குட்பட்டு நெறிப்படுத்தும் ஒரு அமைப்பு தான் நாடு அல்லது அரசு. மனித வாழ்வைப் பொது நிலையில் வரம்புகளுக்குட்பட்டு நிர்ணயிக்கும் ஆட்சி முறை தான் மக்களின், சமூகத்தின் மேன்மைக்கும் உயர்விற்கும் துணை நிற்கின்றது. அது தான் மக்கள் நல அரசாக மிளிர்கிறது. பொதுவாக எந்தவொரு நாடும் அதன் ஆட்சி முறையும் மக்கள் நலனை நோக்கமாகக் கொண்டுதான் செயல்பட வேண்டும். அத்தகைய அறம் சார்ந்த ஆட்சியியல் சிந்தனையான மக்கள் நல அரசு (Welfare State) அமைவதின் அவசியத்தை இரண்டே அடிகளில் சுட்டிக்காட்டிய ஆட்சியியல் வல்லுநர் வள்ளுவராவார் (Varma, 1975).

ஆட்சி முறை எதுவாயினும் நிர்வாகம் செய்திட நிலப்பரப்பு அவசியம். நிர்வாக எல்லைகள் கொண்ட பரப்பை ஆளும் மன்னன் அதனைக் கட்டிக்காப்பதற்கு எத்தகைய பாதுகாப்பு உடைமைகளைப் பெற்றிருக்க வேண்டும் என்பதை இறைமாட்சியின் முதல் குறளான

படைகுடி கூழ்அமைச்சு நட்பரண் ஆறும்

உடையான் அரசருள் ஏறு (குறள் - 381) 
என்பதில் தொகுத்துக் கூறுகின்றார் வள்ளுவர். மக்கள் நல அரசைக் கட்டமைப்பதற்கான இன்றியமையாத பல்வேறு உறுப்புகளைக் கட்டிக் காக்கும் இறை சக்தி கொண்டவனாக மன்னனை மக்கள் கருதினர்.

ஒரு நாடு எத்தகைய தன்மையுடையதாக இருத்தல் வேண்டும்? என்ற வினாவினை எழுப்பி அவ்வினாவிற்கு வள்ளுவர் தரும் பதில் சிறப்பிற்குரியது.

மக்கள் பசிப்பிணியால் வாடக்கூடாது. உடல் நோய்களாலும் வருந்தக் கூடாது. நோயற்ற வாழ்க்கை வாழ வேண்டும். பட்டினியும், பிணியும் ஒரு நாட்டில் ஒழியாவிடில் அந்நாடு வளர்ச்சிப் பாதையில் பயணிக்க இயலாது. எனவே ஒரு நாடு வலிமை மிக்க நாடாக இருத்தல் அவசியம். வெளிப்பகை இல்லாதிருக்க வேண்டுமாயின் மாற்றார் அஞ்சும் வலிமையை ஒரு நாடு பெற்றிருத்தல் வேண்டும் என்பதை

\section{உறுபசியும் ஓவாப் பிணியும் செறுபகையும்}

\section{சேராது இயல்வது நாடு ～(குறள் - 734)}

என்ற குறளின் வழி முன்னிறுத்தும் வள்ளுவர் அதுவே மக்கள் நல அரசிற்கான இலக்கணம் என்றும் சுட்டுகின்றார்.

இவை எல்லாவற்றிற்கும் மேலாக ஒரு நாடு வலிமைமிக்க நாடாக மிளிர்வதற்கு நல்ல ஆட்சியாளன் வேண்டும் என்பதை

\section{ஆங்குஅமைவு எய்தியக் கண்ணும் பயம்இன்றே \\ வேந்துஅமைவு இல்லாத நாடு (குறள் - 740) \\ என்ற குறள் மூலம் வலியுறுத்துகின்றார்.}

\section{ஆட்சியாளரின் தகுதிகள்}

பொதுவாக ஒரு நாட்டின் நிர்வாக முன்னேற்றம் என்பது தனித்திறன் வாய்ந்த, தன்னலம் கருதாத ஆட்சியாளர்கள் ஆட்சி அதிகாரத்தில் அமரும்போது சிறந்து விளங்குகின்றது. இதனை நன்கு உணர்ந்த வள்ளுவர் ஆட்சியாளர்களுக்குத் தனியிடம் தந்துள்ளார்.

நாட்டின் வளர்ச்சியும் தளர்ச்சியும் ஆட்சி செய்வோரின் தகுதிகளையும், குணநலன்களையும் பொறுத்தே அமையும் என்று உறுதிபடக் கூறுகின்றார்.

\section{தூங்காமை கல்வி துணிவுடைமை இம்மூன்றும்}

நீங்கா நிலன்ஆள் பவர்க்கு (குறள் - 383)

என்பது அவர் வாக்கு.

செயலாற்றுவதில் சோர்வின்மை, பரந்து விரிந்த கல்வியறிவு, நன்மை செய்யும் துணிவுடைமை எனும் மூன்று முக்கியத் தகுதிகளை ஆட்சியாளர் பெற்றிருத்தல் அவசியம் என எடுத்துரைக்கின்றார். இம்மூன்று தகுதிகளும் உடையோர் ஆட்சி செய்த போதெல்லாம் நிர்வாகம் சிறப்புற்றிருந்ததுடன் மக்களாட்சி பெருமை பெற்றது. ஆட்சியாளருக்கு இருக்க வேண்டிய அருந் தகுதிகளில் கல்வியை நடுநாயகமாக வைத்தது சிந்திக்கத் தக்கதாகும். மக்கள் நலனுக்குப் பயன்படும் கல்வியைக் கற்றிருத்தல் ஆட்சியாளருக்குப் பெருமை சேர்க்கும். கல்வி கற்ற தகுதிசால் பெருமக்கள் தலைமையில் ஆட்சி நடைபெறுவது நாடு ஏற்றம் பெற வழிவகுக்கும் என அன்றே உணர்த்தியுள்ளார் வள்ளுவர். 


\section{ஆட்சியாளரின் பண்புகள்}

நாடாளும் மன்னன் கோ எனும் பெருமை பெற்றான். இறைவன் மட்டுமல்ல நேர்மையான மன்னன் வாழும் இடமும் கோயில் என்றே பெருமை பெற்றது. முறை பிறழாமல் ஆட்சி செய்த மன்னனை இறைவனுக்கு ஒப்பாகப் போற்றியது பழந்தமிழர் நெறி. தமிழர்கள் மக்கள் நலனே பெரிதெனக் கொண்ட மன்னனிடத்து இறைவனையே கண்டனர்.

ஆள்வோரிடம் பகட்டும் படோடாபமும் ஒரு போதும் கூடாது. மக்களாட்சியில் மக்களிடம் பெறப்படும் வரிப்பணம் அவர்களுக்கே பயன் தரும் திட்டங்களாகப் போய்ச் சேர வேண்டும். ஆட்சி செய்வோர் மக்களின், சமுதாயத்தின் பாதுகாவலர்களே அன்றி வேறிலர். ஆள்பவர்கள் தன்னலம் துறந்த தத்துவ ஞானிகளாக இருத்தல் வேண்டும் என்ற சாக்ரடீசின் கருத்தை ஒப்புநோக்கும் வகையில் ஆள்வோர் மனதில் அழுத்தமாகப் பதிய வேண்டிய பண்புகளை

முறைசெய்து காப்பாற்றும் மன்னவன் மக்கட்கு

இறையென்று வைக்கப் படும் (குறள் - 388)

என்ற குறள் வழி அன்றே வள்ளுவர் உறுதிபடக் கூறியுள்ளார். மன்னன் பிறப்பால் மனிதனானாலும் செய்யும் செயலால் மக்களால் இறைவன் என்று போற்றப்படுவான் என்ற சிந்தனை இன்றைய அரசியல் சூழலை கண் முன் நிறுத்துகின்றது. மக்கள் நலனை முன்னிறுத்தியவர்கள் மீண்டும் ஆட்சிப் பொறுப்பேற்றதையும் புறந்தள்ளியவர்கள் ஆட்சியை இழந்ததையும் உணர்த்துகின்றது.

ஆட்சியாளரின் நிர்வாக வெற்றி என்பது நடத்தைசார் அணுகுமுறைகள், மக்களை வழிநடத்தும் பாங்கு, மக்களுக்கும், ஆட்சியாளருக்கும் இடையிலான உறவு ஆகியவற்றைப் பொறுத்தே அமைகின்றது. இதனை

\section{காட்சிக் கெளியன் கடுஞ்சொல்லன் அல்லனேல்}

மீக்கூறும் மன்னன் நிலம்

என்ற குறளின் மூலம் வலியுறுத்துகின்றார். ஆட்சி நிலைபேறு பெற்றிட ஆட்சியாளர் காண்பதற்கு எளியவனாய், எளிதில் அணுகும் தன்மையுடையவராய், கடுஞ்சொல் கூறாதவனாய், மக்களை அரவணைக்கும் அன்பு நிறைந்தவனாய் இருந்தால் அந்த ஆட்சியை எண்ணிப் பார்த்து உலகம் பெருமையுறும். புகழும். இதன் காரணமாக நாட்டின் ஆட்சிப் பரப்பும் விரிவடையும் என்கிறார் வள்ளுவர்.

\section{ஆட்சியாளரின் செயல்திறம்}

ஆட்சி நிர்வாகம் சிறப்பாகச் செயல்பட வேண்டுமானால் அதற்கு அடிப்படை சீரிய செயல்திறனாகும். அத்தகைய செயல்திறனும் மன உறுதி கொண்டதாக இருத்தல் வேண்டும் என்கிறார் வள்ளுவர்.

\section{வினைத்திட்பம் என்ப தொருவன் மனத்திட்பம்}

மற்றைய எல்லாம் பிற (குறள் - 661)

என்ற குறளின் படி செயல் உறுதி என்பதே மன உறுதி தான் என்று குறிப்பிடுகின்றார். நிறைவேற்ற வேண்டிய செயல் எதுவாயினும் செய்து முடிக்க வேண்டும் என்ற மன உறுதி இல்லையாயின் நிறைவு பெறாது. மன உறுதி சீர்குலையுமானால் செயல்திறனில் தேவையான பாதிப்பை ஏற்படுத்தி நிற்கும். அதனால் செயல்கள் முழுமை பெறாமல் இலக்குகளை அடைய முடியாத நிலை தொடர்ந்து நீடிக்கும். மக்கள் நலன் காக்கும் பொருட்டு ஆட்சியாளர்கள் செயல்பட வேண்டுமானால் அவர்களின் மன உறுதி நிலையானதாக இருத்தல் வேண்டும். மன உறுதி இருந்துவிட்டால் பிற உறுதிகள் அனைத்தும் தானாகவே வந்து சேரும் என்கிறார். 
மேலும் அரசனின் (ஆட்சியாளன்) செயல்பாடுகள் அனைத்தும் பாரபட்ச மற்றதாக, கொடுங்கோல் தன்மையற்றதாக இருத்தல் நலம் எனப் பின்வரும் குறள் மூலம் விளக்குகின்றார் வள்ளுவர்.

\section{துளிஇன்மை ஞாலத்திற்கு எற்றுஅற்றே வேந்தன்}

\section{அளிஇன்மை வாழும் உயிர்க்கு (குறள் - 557)}

மக்களைத் துன்பத்தில் ஆழ்த்தும், அல்லது வருத்தும் தொழிலை மேற்கொண்டு முறையற்ற செயல்களில் ஈடுபடும் ஆட்சியாளர் கொலைத் தொழில் புரிபவரைக் காட்டிலும் கொடியவன் என்று இடித்துரைக்கின்றார் வள்ளுவர்.

\section{தகுந்த பணியாளர்களைத் தேர்வு செய்தல்}

சிறப்பான நிர்வாகத்தை வழங்கும் பொருட்டு தன் கீழ்ப் பணியாற்றும் பணியாளர்களை ஆள்வோர் எங்ஙனம் தேர்வு செய்ய வேண்டும்? என வள்ளுவர் அறுதியிட்டுக் கூறுகின்றார். சொந்த விருப்பு வெறுப்புகளுக்கு அப்பால் தயவு தாட்சண்யமின்றி பொறுப்புடனும் கடமையுணர்வுடனும் மக்கள் நலனை மனதில் கொண்டு பணியாற்றுவோரை அடையாளம் கண்டு பணியமர்த்த வேண்டும். மாறாக, தன் மீது அன்பு கொண்டவர்கள் என்பதற்காக அறிய வேண்டியவற்றை அறியாதவர்களை நம்பித் தேர்வு செய்து அவர்களிடம் அச்செயலை ஒப்படைப்பது மன்னனை அறியாமை இருளில் மூழ்கடிக்கும் என்பதை வள்ளுவர் மிக நுட்பமாக

காதன்மை கந்தா அறிவறியார்த் தேறுதல்

பேதைமை எல்லாம் தரும் (குறள் - 507)

என்ற குறளின் மூலம் வலியுறுத்துகின்றார். மேலும்

அன்புஅறிவு தேற்றம் அவாஇன்மை இந்நான்கும்

நன்குடையான் கட்டே தெளிவு (குறள் - 513)

என்ற குறளில் அன்பு, அறிவு, கலங்காத தெளிவு, ஆசையில்லாமை இந்நான்கு நற்பண்புகளையும் உடையவனைத் தேர்ந்தெடுத்து அவனுக்கான பணியினை ஒப்படைப்பது என்பது மன்னனின் தேர்ந்த நிர்வாகத் திறமைக்குச் சான்றாகும் என அறிவுறுத்துகின்றார் (Mohanaraju, 2010; Varadharasan 2004).

\section{அமைச்சர்}

மக்கள் நல அரசில் நிர்வாகம் சீரிய முறையில் நடைபெற அமைச்சர்களுக்குத் தனிப் பங்கு உண்டு. தன் கூர்மதியால் தகுந்த நேரத்தில் தக்க ஆலோசனைகளை வழங்கி ஆட்சி மற்றும் ஆட்சியாளர் மீதான அவப்பெயரை துடைத்தெறிந்து ஆட்சிச் செங்கோல் சாய்ந்து விடாமல் தூக்கிப் பிடிப்பதில் அமைச்சருக்கு நிகர் அவரே என்பதை வள்ளுவர்

கருவியும் காலமும் செய்கையும் செய்யும்

அருவினையும் மாண்டது அமைச்சு (குறள் - 631)

என்ற குறளின் வழி குறிப்பால் உணர்த்துகிறார்.

அத்தகைய பெருமை வாய்ந்த அமைச்சன் பெற்றிருக்க வேண்டிய குணநலன்களைக் கீழ்க்காணும் குறளில் எடுத்துரைக்கிறார்.

அறன்அறிந்து ஆன்றுஅமைந்த சொல்லான்எஞ் ஞான்றும்

திறன்அறிந்தான் தேர்ச்சித் துணை (குறள் - 635) 
அறம் அறிந்தவனாய், அறிவு நிறை சொல்லை உடையவனாய், எக்காலத்திலும் செயல் செய்யும் திறனறிந்தவனாய் உள்ளவனே ஆராய்ந்து கூறும் அமைச்சனே மன்னுக்குச் சிறந்த அருந்துணையாவான்.

\section{ஆட்சியாளரின் நீதிமுறை}

பொதுவாக நீதி வழங்குதல் என்பது ஒரு பிரச்சினையை நெறிமுறைகள் தவறாது நடுநிலைத் தன்மையுடன் அணுகி தக்கதொரு தீர்வினை வழங்குவதாகும். ஆட்சி அதிகாரத்தைக் கையில் வைத்துள்ள ஆட்சியாளர்கள் நடுநிலை பிறழாது நீதி வழங்கி ஆட்சி முறைமைக்குச் சிறப்புச் சேர்க்க வேண்டும் என்று வள்ளுவர் வலியுறுத்துகின்றார்.

\section{தக்காங்கு நாடித் தலைச்செல்லா வண்ணத்தால்}

\section{ஒத்தாங்கு ஒறுப்பது வேந்து}

மேலும் நடைபெற்ற குற்ற நிகழ்வை அல்லது பிரச்சினையை முறைப்படி தீர ஆராய்ந்து அதற்குப் பொருந்தும் வகையிலும் மீண்டும் அக்குற்றச் செயல்களில் ஈடுபடாதவாறும் தகுந்த தண்டனை வழங்க வேண்டும். அத்தண்டனை வழங்கலும் சிபி, மனுநீதிச் சோழன் போன்று அறம் வழுவாது இருத்தல் வேண்டும் என்கிறார் (Rajaram, 2015; Drew \& Lazarus 2007).

எல்லாவற்றிற்கும் மேல் சட்டம், நீதிமுறைகள், நீதிமன்றங்கள், தண்டனைகள் குற்றமிழைத்தோரைத் திருத்தவேயன்றி தண்டிப்பதற்காக அல்ல எனும் சமகாலச் சிந்தனையை வள்ளுவர்

\section{கடிதோச்சி மெல்ல எறிக நெடிதாக்கம்}

\section{நீங்காமை வேண்டு பவர் (குறள் - 562)}

என்று வலியுறுத்துகின்றார். அதாவது கடுமையாகத் தண்டிப்பவன் போலத் தன்னைக் காட்டிக் கொண்டாலும் வரம்பு மீறாமல் அவன் மனம் திருந்தும் வகையில் தண்டிப்பது நீதி வழங்கும் முறையிலும் அறத்தை நிலைநாட்டும் உயர் பண்பாகும். அவ்வாறு செயல்படும் மன்னனின் செல்வாக்கும் ஆட்சியும் நெடுங்காலம் நீடிக்கும் என்கிறார்.

\section{ஆட்சி முறை}

உலகில் அறம் தழைக்கவும், அறம் வளர்க்கும் சான்றோர் வாழ்வு சிறக்கவும் அடிப்படையாக அமைவது ஆட்சிமுறையாகும். ஆட்சி முறைக்கு அடையாளமாக இருப்பது அம்மன்னின் செங்கோல் தான் என்ற கருத்தும் நிலவுகின்றது. செங்கோல் என்பது எதற்கும் வளையாத, நேர்மையான ஆட்சியைக் குறிக்கும். குடிமக்களின் உயிரையும் உடைமையையும் பாதுகாக்கும் ஆட்சியியல் அறத்தைப் பேணுவது ஆட்சியாளரது தலையாயக் கடமையாகும். ஆட்சியில் உருவாகும் குறை, நிறைகளைச் சீர்தூக்கிப் பார்த்துச் செம்மையாக ஆள்வதே நல்லாட்சியாகும். இதுவே செங்கோல் வழுவாத, நெறிபிறழாத ஆட்சிமுறையைக் குறிக்கும்.

\section{வான்நோக்கி வாழும் உலகெல்லாம் மன்னவன்}

\section{கோல்நோக்கி வாழும் குடி (குறள் - 542)}

என்ற குறளில் மழையை உதாரணமாக்கி, உலக உயிர்களனைத்தும் மழையை எதிர்பார்த்து வாழ்வதைப் போல் மக்களாட்சியில் மக்கள் அனைவரும் ஆட்சியாளரின் செங்கோலை நோக்கி வாழ்கின்றனர். மண்ணில் உள்ள அனைவரையும் பாரபட்சமின்றி வாழவைக்கும் மழைக்கு நிகராக அரசனின் (ஆட்சியாளன்) செங்கோலைப் பேசுகின்றார்.

மழை எப்படி மக்களை விருப்பு வெறுப்பின்றி வாழவைக்கின்றதோ அதுபோல மக்களுக்கு நெறிமுறை தவறாத, நடுநிலைமையுடன் கூடிய நேர்மையான ஆட்சியை வழங்கி வாழவைப்பதே 
ஆட்சியாளரின் பணி என்பதைவிட அதுவே அவன் மீதான நம்பிக்கையை மக்கள் மனதில் நிலைநிறுத்தும் என்பது வள்ளுவச் சிந்தனை.

\section{குடிதழீஇக் கோலோச்சும் மாநில மன்னன்}

\section{அடிதழீஇ நிற்கும் உலகு (குறள் - 544)}

என்னும் குறளும் இன்றைய மக்களாட்சியின் சிறப்புத் தன்மைகளை காலம் கடந்தும் பொதுமை நோக்கில் புலப்படுத்தி நிற்கின்றது. மக்கள் கருத்தை மதித்து அவர்களை அன்புடன் அரவணைத்து அறிவு சார்ந்த புரிதலை ஏற்படுத்தி சூழ்நிலை அறிந்து ஆட்சி செய்தலே குடி தழுவிக் கோலோச்சுவதாகும். அத்தகைய ஆட்சியாளனின் அடியொற்றி அரசின் வழி நிற்பர் மக்கள். இது ஆட்சியாளருக்கும் மக்களுக்குமான உறவைப் பலப்படுத்துவதுடன் நல்லாட்சிக்கான நற்சிந்தனையை ஆட்சியாளரின் மனதில் விதைத்து நிற்கின்றது.

\section{ஆட்சியாளரின் மதிநுட்பம்}

உளவு பார்த்தல் மற்றும் புலனாய்வு மூலம் ஆட்சிக்கெதிரான, ஆள்வோருக்கெதிரான செய்திகளை, நிகழ்வுகளை அவ்வப்போது அறிவிப்பது ஆட்சிப் பாதுகாப்பிற்கு அவசியமான ஒன்றாகும். இந்த இரகசியப் பணிகளை முடியாட்சிக் காலத்தில் மன்னர்கள் நம்பிக்கை கொண்ட ஒற்றர்கள் வழி மேற்கொண்டனர்.

ஆட்சியாளர்கள் எந்தவொரு செய்தியையும், நிகழ்வையும் எளிதில் நம்பிவிடுதல் கூடாது. எவரையும் முழுமையாக நம்பி விடுதல் கூடாது. அதற்காக அனைவரையும் சந்தேகப்பார்வையோடும் பார்த்தல் கூடாது. தேர்ந்த ஒற்றர்கள் மூலம் சரியான தகவல்களின்படி உண்மையைக் கண்டறிதல் வேண்டும். பெற்ற தகவல்களின் உண்மைத் தன்மையை முழுமையாக உணர்ந்து, ஆராய்ந்து அறிதல் வேண்டும். முழுமையாக ஆராய்ந்து, உண்மையை உணராது போனதால் தான் பாண்டிய நெடுந்செழியன் பழிக்கு ஆளாகினான்.

\section{ஒற்றுஒற்றித் தந்த பொருளையும் மற்றும்ஓர்}

\section{ஒற்றினால் ஒற்றிக் கொளல் (குறள் - 588)}

என்கின்றார் வள்ளுவர். அதாவது நம்பிக்கைமிகு ஒற்றனாயினும் அவனை அறியாமல் கூட பிழை செய்துவிடலாம். எனவே ஓர் ஒற்றன் தரும் தகவலை பிறிதொரு ஒற்றன் வாயிலாக உண்மையை உறுதி செய்து கொள்ளும் வகையில் ஆட்சியாளருக்குப் பகுத்தறிந்து நோக்கும் மதிநுட்பம் வேண்டும் எனச் சுட்டுகின்றார். ஆட்சியியல் கலையை சாதாரணமானதாக எண்ணாமல் அதனை மிகுந்த நுட்பத்துடன் அணுகி உணர்ந்து கூறுகின்றார் என்பதற்கு இக்குறளே சான்று. இன்று மட்டுமல்ல என்றும் ஆட்சியாளர்களுக்கு இக்குறள் வழிகாட்டும்.

\section{முடிவுரை}

ஆட்சியியல் நெறிமுறைகள், ஆட்சியாளரின் சிறப்புகள் போன்றவற்றைச் சரியாக எடுத்துக் கூறினாலும் தவறான ஆட்சியியல் நடவடிக்கைகளால் ஏற்படும் விளைவுகளையும், அதனால் சமூகத்தில் ஏற்படும் வாழ்வியல் மதிப்படடுகளின் மீீதான தாக்கங்களையும் சுட்டிக்காட்டும் வள்ளுவர் ஆட்சி நல்லாட்சியாக மாறுவதற்கான வழிகளையும் குறிப்பிடுகின்றார். திருக்குறள் நீதி நூல், அறநூல், பொருளாதார வழிகாட்டி நூல், தத்துவ நூல், அரசியல் நெறியுரை நூல் எனப் பல்வேறு வகையாகப் போற்றப்படினும் எல்லாவற்றையும் விட திருக்குறள் மனித வாழ்க்கை மாண்புற உரிய வழிகளைக் காட்டியதால் அது ஒரு வாழ்வியல் நூலாகப் போற்றப்படுவது தான் மிகவும் பொருந்தும். வள்ளுவர் சுட்டும் அறம் சார்ந்த அரசியலை முன்னெடுப்பதும் மக்கள் நலனை மட்டுமே குறிக்கோளாகக் கொண்டு செயல்படும் அரசை நிர்மாணிப்பதும் இன்றைய சூழலில் சாத்தியமா? என்ற கேள்வி எழுகின்றது. குறள் கூறும் கோட்பாடுகளைப் பின்பற்றி நேர்கோட்டில் ஆட்சி நடத்துவது என்பது தற்போதைய சுயநலச் 
சமூகத்தில் இயலாத காரியம் தான். ஆனால் அறநெறிக்கொள்கைகளை இலக்காகக் கொண்டு இயங்கும் அரசியல் மற்றும் ஆட்சியியல் ஆளுமைகள் இருந்தால் சாத்தியம் தான். கட்டமைக்கப்பட்ட ஆட்சி முறைமைகளைப் பயன்படுத்தி அறவாழ்க்கையை, ஆட்சியை எவ்வாறு வழங்கிட வேண்டும் என்பதைப் புலப்படுத்துவதே வள்ளுவ ஆட்சியியல் நெறியாகும். மக்கள் நலனை இலக்காகக் கொண்டு ஆட்சியாளர்கள் ஆட்சிபுரிதல் அவசியமாகின்றது. இரண்டாயிரம் ஆண்டுகளுக்கு முன்பு வள்ளுவர் கூறிய மக்கள் நல அரசு அமைவதற்கான ஆட்சியியல் சிந்தனைகள் எக்காலத்திற்கும் எந்த அமைப்பிற்கும் பொருந்துவதால் அவரை ஒரு சிறந்த ஆட்சியியல் வல்லுநராகவே பறைசாற்றுகின்றது என்றால் அது மிகையல்ல.

\section{References}

Drew W.H., Lazarus, J., (2007) Thiruvalluvar. Thirukkural, (Trans.), Asian Educational Services, New Delhi, India.

Mohanaraju, K., (2010) Thirukkural: Makkal Vurai, Manivasakar Pathippagam, Chennai, India.

Perumal, C.A., (1974) The Political Clarity of Tirukkural, Madurai Kamaraj University, Madurai, India.

Pope, G.U., Drew, W.H., Lazarus J., Ellis, F.W., (1982) Thirukkural: English Translation and Commentary, The South India Saiva Siddhantha Works Publishing Society, Tinnevelly, India.

Rajaram, M., (2015) Glory of Thirukkural, International Institute of Tamil Studies, Chennai, India.

Sankaravallinayagam, A., (2003) Pulaththurai Mutriya Poyyil Pulavar, Agaram, Thanjavur, India.

Thangayyan, C., (1950) Sangam Political Philosophy, The Indian Journal of Political Science, 11(2) 52-60.

Varadharasan, Mu., (2004) Thirukkural Thelivurai, The South India Saiva Siddhantha Works Publishing Society, Tinnevelly, India.

Varma, S.P., (1975) Modern Political Theories, Vikash Publishing House Pvt. Ltd., New Delhi, India.

Funding: NIL

Acknowledgement: NIL

Conflict of Interest: NIL

About the License:

\section{(c) (1)}

Attribution 4.0 International (CC BY 4.0)
(C) The author 2021. The text of this article is licensed under a Creative Commons Attribution 4.0 International License 